\title{
Role of Nitric Oxide Signaling in Endothelial Differentiation of Embryonic Stem Cells
}

\author{
Ngan F. Huang, Felix Fleissner, John Sun, and John P. Cooke
}

Signaling pathways that govern embryonic stem cell (ESCs) differentiation are not well characterized. Nitric oxide (NO) is a potent vasodilator that modulates other signaling pathways in part by activating soluble guanylyl cyclase (sGC) to produce cyclic guanosine monophosphate (cGMP). Because of its importance in endothelial cell (EC) growth in the adult, we hypothesized that NO may play a critical role in EC development. Accordingly, we assessed the role of NO in ESC differentiation into ECs. Murine ESCs differentiated in the presence of NO synthase (NOS) inhibitor NG-nitroarginine methyl ester (L-NAME) for up to 11 days were not significantly different from vehicle-treated cells in EC markers. However, by 14 days, L-NAME-treated cells manifested modest reduction in EC markers CD144, FLK1, and endothelial NOS. ESC-derived ECs generated in the presence of L-NAME exhibited reduced tube-like formation in Matrigel. To understand the discrepancy between early and late effects of L-NAME, we assessed the NOS machinery and observed low mRNA expression of NOS and sGC subunits in ESCs, compared to differentiating cells after 14 days. In response to NO donors or activation of NOS or sGC, cellular cGMP levels were undetectable in undifferentiated ESCs, at low levels on day 7, and robustly increased in day 14 cells. Production of cGMP upon NOS activation at day 14 was inhibited by L-NAME, confirming endogenous NO dependence. Our data suggest that NOS elements are present in ESCs but inactive until later stages of differentiation, during which period NOS inhibition reduces expression of EC markers and impairs angiogenic function.

\section{Introduction}

P LURIPOTENT STEM CELLS SUCH as embryonic stem cells (ESCs) have therapeutic potential for regenerative medicine because they have unlimited self-renewal capacity and can give rise to lineages comprising all 3 germ layers [1,2]. As a result, ESCs are regarded as a potential unlimited source of therapeutic cells to replace diseased tissues. With respect to cardiovascular diseases, ESCs have been successfully differentiated into cardiovascular lineages such as endothelial cells (ECs), cardiomyocytes, and vascular smooth muscle cells, and have been shown to have functional benefit in preclinical models of myocardial infarction and peripheral arterial disease [3-6].

However, the signaling pathways that regulate ESC differentiation are not well characterized. Among the various important signaling molecules is nitric oxide (NO), a short-lived free radical that is produced by nitric oxide synthase (NOS) upon oxidation of L-arginine [7]. The biological effects of $\mathrm{NO}$ are in part mediated by its activation of soluble guanylate cyclase (sGC) to produce cyclic guanosine monophosphate (cGMP). Subsequently, cGMP modulates the activity of cGMP-dependent protein kinases, phosphodiesterases, and ion channels [8]. In this way, NO regulates diverse cellular processes in the cardiovascular, central nervous system, and immune system of the adult mammal. Several reports also suggest a role for $\mathrm{NO}$ signaling in cardiac differentiation [9-12]. Furthermore, defects in bicuspid aortic valves and atrial and ventricular septa are common in endothelial NOS (eNOS)-deficient neonates [13,14]. However, little is known regarding the role of $\mathrm{NO}$ in ESC differentiation to ECs. In the adult mammal, we and others have shown that NO is a potent mitogen and survival factor for ECs, as well as an angiogenic agent [15-17]. Because of the important role NO plays in maintaining EC growth in adults, we hypothesized that it may also play a critical role in endothelial development.

Here, we report the differential expression of NOsignaling components during endothelial differentiation and the effect of NOS inhibition on endothelial differentiation. 


\section{Materials and Methods}

\section{ESC culture and differentiation}

Murine ESCs (D3; ATCC, Manassas, VA) were routinely cultured on mitotically inactivated mouse embryonic fibroblasts (MEF) in growth media containing leukemia inhibitory factor as described [18]. For differentiation studies, ESCs were dissociated by trypsin and then pre-plated onto gelatin-coated dishes for $60 \mathrm{~min}$ in growth media at $37^{\circ} \mathrm{C}$ to deplete residual MEFs. Next, $10^{6}$ ESCs were transferred to ultra-low nonadhesive dishes to initiate embryoid body (EB) formation in an endothelial differentiation media that consisted of $\alpha$-minimum Eagle's media, 10\% fetal bovine serum (FBS), 1\% penicillin/streptomycin, and $0.05 \mathrm{mM}$ $\beta$-mercaptoethanol (Sigma, St. Louis, MO) [12]. After 4 days of suspension culture, the EBs were reattached onto $0.2 \%$ gelatin-coated dishes and cultured in differentiation media. Where specified, cells were treated with differentiation media supplemented by NOS inhibitor NG-nitroarginine methyl ester (L-NAME, $10^{-5} \mathrm{M}$ or $10^{-4} \mathrm{M}$; Sigma) or dimethyl sulfoxide (DMSO) as vehicle control. The media was changed daily, and at indicated time points, samples were assayed for gene expression, protein expression, Matrigel tube-like formation assay, or cGMP activity.

\section{Quantitative polymerase chain reaction}

Samples were lysed in Trizol (Invitrogen, Carlsbad, CA), and total RNA was isolated using the RNEasy Kit (Qiagen, Valencia, CA). Using the Superscript II reverse transcriptase (Invitrogen), first strand cDNA was synthesized according to the manufacturer's instruction. Primers for Taqman qPCR assays consisted of FLK1, CD144 (VE-cadherin), von Willebrand factor (VWF), eNOS, soluble guanylyl cyclase (sGC) subunits $\alpha_{2}, s G C \alpha_{3}$ (formerly named $s G C \alpha_{1}$ ), $s G C \beta_{2}$, $\mathrm{sGC} \beta_{3}$ (formerly named $s \mathrm{GC} \beta_{1}$ ), dimethylarginine dimethylaminohydrolase $(D D A H) 1$, and $D D A H 2$ (all from Applied Biosystems, Foster City, CA). The $18 \mathrm{~S}$ primers were obtained from Shetzline et al. [19]. qPCRs were performed on a 7300 Real-Time PCR system (Applied Biosystems) for 40 cycles. The data were assessed by the $\Delta \Delta \mathrm{Ct}$ method [20], normalized to $18 \mathrm{~S}$ housekeeping gene, and then expressed as relative fold changes $(n=3)$.

\section{Immunoblotting}

At indicated time points, samples were lysed in RIPA buffer (Pierce, Rockford, IL) containing protease and phosphatase inhibitors and quantified for total protein quantity by the bicinchoninic (acid) BCA protein assay (Pierce). Equally loaded protein samples were resolved by sodium dodecyl sulfate polyacrylamide gel electrophoresis (SDS-PAGE) and then transferred onto nitrocellulose membranes (Invitrogen, Carlsbad, CA). The membranes were blocked in 5\% bovine serum albumin (BSA) before incubating with the following antibodies: FLK1 (Cell Signaling Technology, Danvers, MA), eNOS (BD Biosciences, San Diego, CA), or $\alpha$-tubulin (Cell Signaling Technology). Horse radish peroxidase (HRP)conjugated secondary antibodies were applied, and the proteins were visualized by an ECL Detection Kit (Amersham, Piscataway, NJ). Quantification of protein quantities were carried out by normalizing to $\alpha$-tubulin and are expressed in relative fold change $(n=3)$.

\section{Immunofluorescence staining}

Samples were fixed in $4 \%$ paraformaldehyde and immunofluorescently stained, as described previously [21], for the antibodies $\mathrm{sGC} \alpha_{3}$ (Sigma, St. Louis, MO), sGC $\beta_{3}$ (Sigma), eNOS, or CD144 (BD Biosciences, San Diego, CA). Total nuclei were stained by Hoechst 33342 (Invitrogen, Carlsbad, CA) dye before imaging on a fluorescent microscope (Nikon, Burlingame, CA), and images were captured with a SPOT RT color camera (Diagnostic Instruments, Sterling Heights, $\mathrm{MI})$.

\section{cGMP activity}

To investigate the contribution of endogenous or exogenous NO release at various time points of differentiation, cells were incubated for $30 \mathrm{~min}$ with Dulbecco's modified Eagle's medium (DMEM) containing calcium ionophore A23187 $\left(2 \times 10^{-5}\right.$ M; Sigma), BAY-41-2272 (BAY41, $10^{-5} \mathrm{M}$; EMD Chemicals, Gibbstown, NJ), ( \pm )-S-Nitroso-Nacetylpenicillamine (SNAP, $10^{-4} \mathrm{M}$, EMD Chemicals), or DMSO vehicle control. In addition, to assess the effect of L-NAME treatment on cGMP activity, after 14 days of differentiation in the presence or absence of L-NAME $\left(10^{-5} \mathrm{M}\right)$, cells were incubated with DMEM containing one of the following treatments: (1) A23187 $\left(2 \times 10^{-5} \mathrm{M}\right)$, (2) BAY41 $\left(10^{-5}\right.$ M), (3) SNAP $\left(10^{-4} \mathrm{M}\right)$, (4) BAY41+SNAP, (5) BAY41+A23187, or (6) DMSO vehicle control (Basal). All media were further supplemented with the cGMP phosphodiesterase inhibitor isobutylmethylxanthine (IBMX, 10 ${ }^{-3} \mathrm{M}$; Sigma). After $30 \mathrm{~min}$, the cells were lysed in $0.1 \mathrm{~N} \mathrm{HCl}$ and assayed for cGMP activity as described by the manufacturer (Cayman Chemical, Ann Arbor, MI). For each sample, cGMP activity was normalized to the abundance of total protein, as assessed by BCA protein quantification assay $(n=3)$.

\section{Fluorescently activated cell sorting (FACS) and Matrigel tube-like formation}

After 3 weeks of differentiation in L-NAME $\left(10^{-4} \mathrm{M}\right.$ or $10^{-5}$ M), ESC-derived endothelial cells (ESC-ECs) were purified by FACS. Cells were dissociated using dissociation buffer (Invitrogen, Carlsbad, CA), blocked in 10\% normal mouse serum, and then incubated with anti-CD144 antibody (BD Biosciences, San Diego, CA), followed by allophycocyanin (APC)-conjugated secondary antibody. As a negative control, immunoglobulin $\mathrm{G}$ isotype was used in replacement of primary antibody. The cells were purified using FACS Vantage (BD Biosciences). To perform the Matrigel tube-like formation assay, purified ESC-ECs derived from L-NAME treatment were seeded on growth factor-reduced Matrigel at a density of $1 \times 10^{5}$ cells/well in differentiation media without L-NAME. After $24 \mathrm{~h}$, the cells were fixed in paraformaldehyde $(4 \%)$. Phase-contrast images were taken under $10 \times$ objectives, and the number of branch points was quantified by NIH ImageJ software version 1.41 o $(n=4)$ [22].

\section{Statistical analysis}

All data are expressed as mean \pm standard deviation. Statistical analysis was performed by the Student's $t$-test for comparison of 2 groups or one-way analysis of variance (ANOVA) with Holm's adjustment for multiple comparisons. 
Repeated measurements of samples over time were analyzed by repeated measures ANOVA with Holm's adjustment. Statistical significance was accepted at $P<0.05$.

\section{Results}

\section{Effect of L-NAME on endothelial differentiation of ESCs}

To investigate the role of L-NAME on endothelial differentiation of ESCs, we treated differentiating ESCs with L-NAME for 14 days and carried out qPCR of endothelial genes. After 14 days of differentiation in the presence of L-NAME, the mature endothelial marker CD144 was significantly inhibited by $34 \%$ in the presence of L-NAME $\left(10^{-5}\right.$ $\mathrm{M})$, when compared to the control group $(P<0.01)$ (Fig. 1A). L-NAME $\left(10^{-5} \mathrm{M}\right.$ or $\left.10^{-4} \mathrm{M}\right)$ treatment also significantly down-regulated eNOS expression by $50 \%$ or $52 \%$, respectively $(P<0.01)$ (Fig. 1B). Similarly, the endothelial progenitor marker, FLK1, was significantly down-regulated by $34 \%$ or $33 \%$, respectively, after treatment with L-NAME $\left(10^{-5} \mathrm{M}\right.$ or $\left.10^{-4} \mathrm{M}\right)$ for 14 days $(P<0.01)$ (Fig. $\left.1 \mathrm{C}\right)$. These observations at the mRNA level were further substantiated by immunoblots after 14 days of differentiation, demonstrating a significant inhibition of eNOS and FLK1 at the protein level with L-NAME $\left(10^{-4}\right.$ M; Fig. 1D-1F).

\section{Effect of L-NAME on Matrigel tube-like formation by ESC-ECs}

To further explore the inhibitory effects of L-NAME on endothelial differentiation, we questioned whether chronic treatment of L-NAME could impair endothelial function in the ESC-ECs. After 3 weeks of culture in the presence of L-NAME $\left(10^{-5} \mathrm{M}\right.$ or $\left.10^{-4} \mathrm{M}\right)$ or DMSO vehicle control, the ESC-ECs were purified by the positive expression of CD144. As shown in Figure 2B, the percentage of CD144 ${ }^{+}$cells in the group treated with L-NAME $\left(10^{-5} \mathrm{M}\right)$ was $\sim 20 \%$, and not significantly different from the percentage of cells in other treatment groups (data not shown). After purification based on positive expression of CD144, the cells were further
A

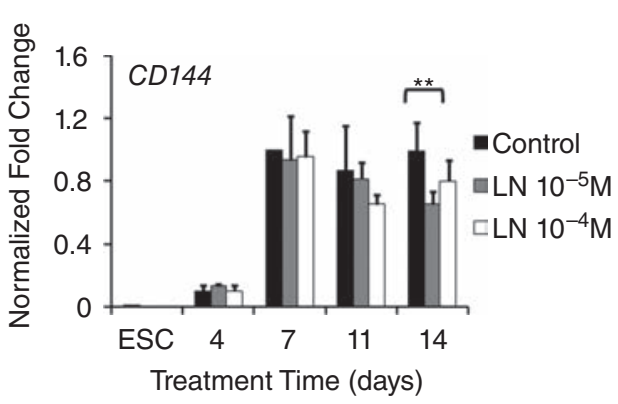

B

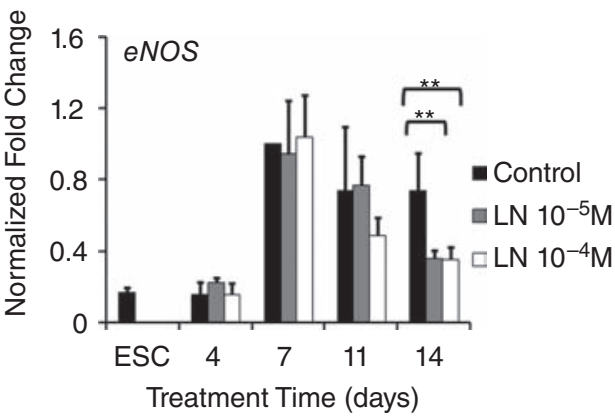

C

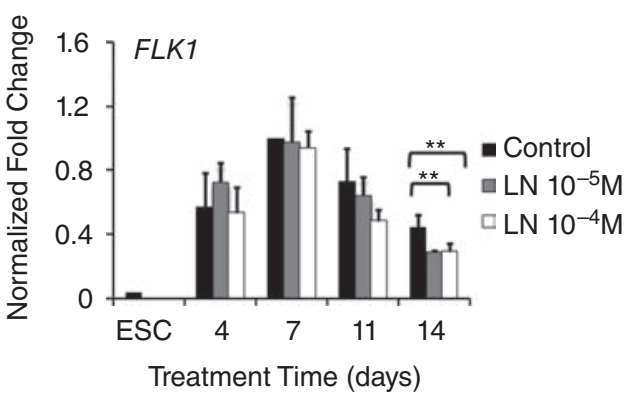

D

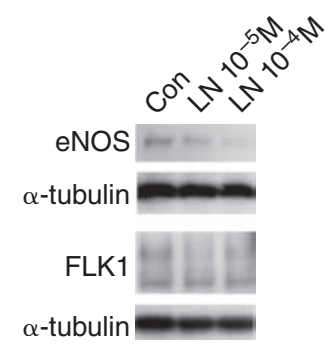

E

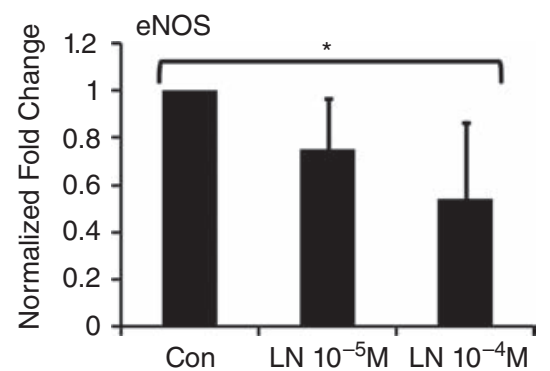

$\mathbf{F}$

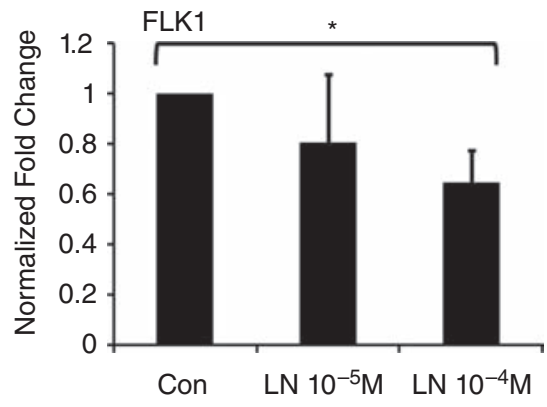

FIG. 1. Effect of NG-nitroarginine methyl ester (L-NAME) on endothelial differentiation of embryonic stem cells (ESCs). (A) Gene expression time course of endothelial differentiation in the absence (Con) or presence of L-NAME (LN, $10^{-5} \mathrm{M}$ or $10^{-4}$ M) for CD144, (B) endothelial nitric oxide synthase (eNOS), and (C) FLK1. Data normalized to 18S and expressed as relative fold change. (D) Immunoblots for endothelial markers 14 days after differentiation in the presence of L-NAME. Quantification of immunoblots for $(\mathbf{E})$ eNOS and $(\mathbf{F})$ FLK1 $(n=3)$. Statistically significant comparisons $\left({ }^{*} P<0.05\right.$; $\left.{ }^{* *} P<0.01\right)$. 
FIG. 2. Purification and characterization of embryonic stem cell-derived endothelial cells (ESC-ECs). The ESCs were differentiated for 3 weeks and then purified by fluorescently activated cell sorting (FACS) based on positive expression of the mature endothelial marker, CD144. Shown are the (A) isotype negative control and (B) $\mathrm{CD}_{144^{+}}$population. After purification, the $\mathrm{CD} 144^{+}$cells were characterized by immunofluorescence staining for (C) CD144 or (D) endothelial nitric oxide synthase (eNOS) to confirm their endothelial phenotype. Scale bar $=25 \mu \mathrm{m}$. Color images available online at www.liebertonline.com/scd.
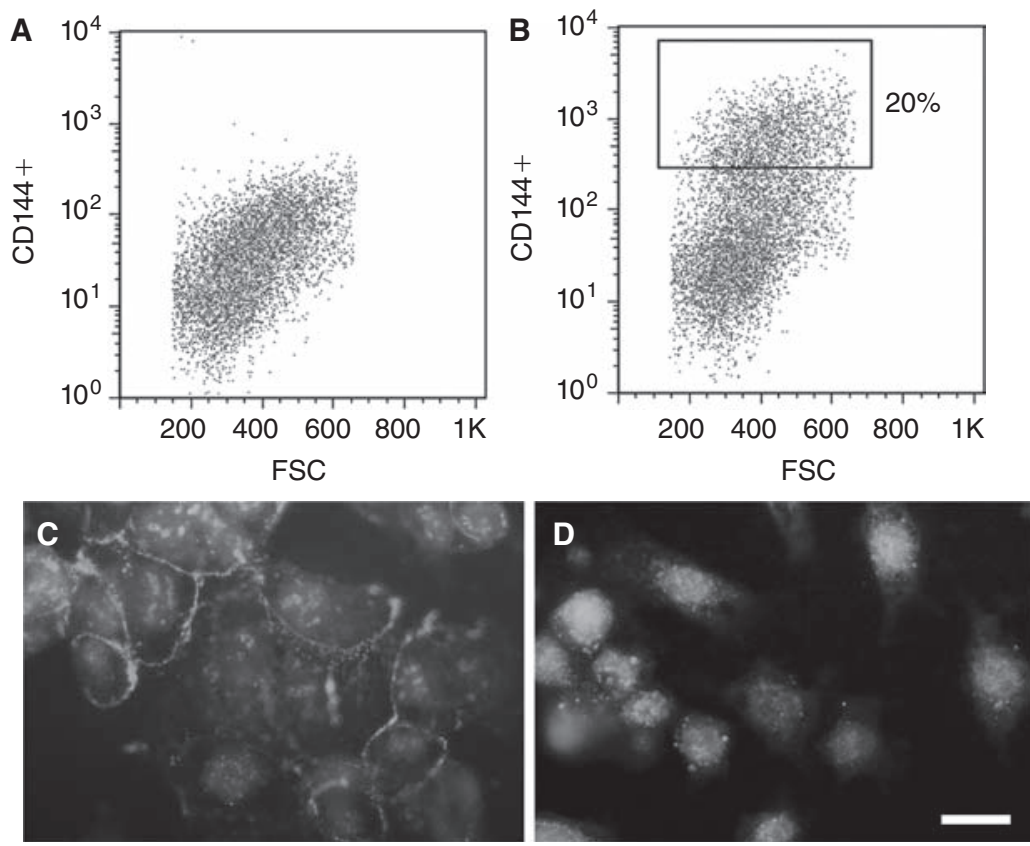

characterized by immunofluorescence staining of CD144 and eNOS to confirm their endothelial phenotype (Fig. 2C and 2D). We noted that cells previously exposed to L-NAME had alterations in function, even after L-NAME withdrawal. Although the percentages of CD144-expressing cells were similar for all groups (20\%), the cells treated for 21 days with L-NAME $\left(10^{-5} \mathrm{M}\right.$ dose only) appeared to have impairment in function, with reduced capacity (by $25 \%$ ) to form tubelike structures in Matrigel, even in the absence of L-NAME (Fig. 3, $P<0.05$ ). This result demonstrated that chronic treatment of L-NAME during endothelial differentiation of ESCs could impair an important endothelial function.

\section{Temporal expression of sGC and DDAH during endothelial differentiation}

To explore the mechanism of by which L-NAME affected late but not early expression of EC markers, we surveyed $\mathrm{NO}$-signaling components during the course of endothelial differentiation for up to 2 weeks. Using qPCR, we examined the mRNA expression of $s G C$ subunits $\left(\alpha_{2}, \alpha_{3}, \beta_{2}\right.$, and $\left.\beta_{3}\right)$ as well as DDAH1 and DDAH2. The mRNA abundance was normalized to $18 \mathrm{~S}$ housekeeping gene and expressed as fold changes relative to the undifferentiated ESC group. As shown in Figure $4 \mathrm{~A}-4 \mathrm{D}$, mRNA transcripts could be detected in ESCs for all $s G C$ subunits, although the relative abundance of $s G C \alpha_{3}$ was particularly low. During the course of differentiation, the mRNA levels increased significantly for each subunit, namely a 100-fold increase in $s G C \alpha_{3}$ and a 10 -fold increase for $s G C$ subunits $\alpha_{2}, \beta_{2}$, and $\beta_{3}$. The gene expression pattern was similar for $D D A H 1$ and $D D A H 2$, showing a significant increase with differentiation time (Fig. $4 \mathrm{E}$ and $4 \mathrm{~F}, P$ $<0.05)$. The time course of gene expression was further supported by immunofluorescence staining showing that the increased intensity of $\mathrm{sGC} \alpha_{3}$ and $\mathrm{sGC} \beta_{3}$ expression as ESCs differentiated through day 14 (Fig. 5A and 5B). The increased expression of $\mathrm{sGC} \alpha_{3}$ and $\mathrm{sGC} \beta_{3}$ was associated in part by the emergenceofESC-ECs, asCD144-expressing cellsco-localized with $\mathrm{sGC} \alpha_{3}$ and $\mathrm{sGC} \beta_{3}$ at day 14 of differentiation. However, $\mathrm{sGC} \alpha_{3}$ and $\mathrm{sGC} \beta_{3}$ were also expressed in other cell types besides ESC-ECs, which is consistent with literature indicating that sGCs are expressed in numerous mammalian tissues [23]. These results indicated that NO-signaling components are present in ESCs, but are up-regulated during the course of ESC differentiation. The up-regulation of NO-signaling components with differentiation time is consistent with the observed effect of L-NAME on EC markers at later stages of differentiation.

\section{Production of cGMP activity during endothelial differentiation}

To determine whether the differential gene expression of NO-signaling components reflected the ability of the cells to produce cGMP in response to NO stimulation, we stimulated ESCs or differentiating cells (7 or 14 days) with A23187, BAY41, SNAP, or DMSO vehicle control. The calcium ionophore A23187 increases intracellular calcium levels to increase NOS activity, and thereby increases endogenous NO activation of sGC. SNAP is an exogenous NO donor and mimics endogenous NO. In contrast, BAY41 activates sGC in an NO-independent manner and can potentiate the effect of NO to increase cGMP levels [8]. As shown in Figure 6A-6D, ESCs did not produce detectable cGMP after the addition of any of these agents. Basal levels of cGMP were detectable by 7 days, and by day 14 had increased to $4.4 \pm 0.1 \mathrm{pmol} /$ mg protein (Fig. 6A). A response to A23187 was also observed at day 7 and was greater at day $14(8.7 \pm 1.3 \mathrm{pmol} /$ mg protein $(P<0.05$, Fig. $6 \mathrm{~B})$. Similarly, SNAP and BAY41 were potent stimulators of $\mathrm{SGC}$, with a greater response observed after 14 days of differentiation (362.8 $\pm 19.31 \mathrm{pmol} /$ $\mathrm{mg}$ and $205.0 \pm 15.5 \mathrm{pmol} / \mathrm{mg}$, respectively, $P<0.05$, Fig. $6 \mathrm{C}$ and 6D). Together, these data indicate that even though components of the NOS pathway are detectable, ESCs are not capable of generating CGMP via this pathway. Furthermore, as ESCs differentiate, the elements of the NOS pathway are 


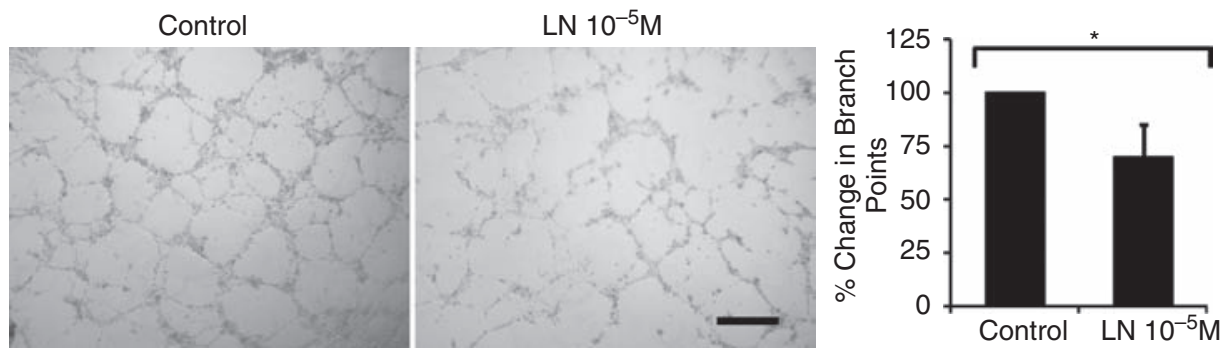

FIG. 3. Effect of NG-nitroarginine methyl ester (L-NAME) treatment on tube-like formation of embryonic stem cell-derived endothelial cells (ESC-ECs) in Matrigel. Endothelial differentiation of ESCs was carried out in the presence of L-NAME (LN, $10^{-5}$ $\mathrm{M}$ or $10^{-4} \mathrm{M}$ ) for 21 days. After purification by CD144 ${ }^{+}$expression, the ESC-ECs were assessed for tube-like formation upon withdrawal of L-NAME for $24 \mathrm{~h}$. Representative images of tube-like formation for cells having undergone chronic L-NAME or dimethyl sulfoxide (DMSO) vehicle control treatment are shown along with quantification of branch points $(n=4)$. Prior exposure to L-NAME $\left(10^{-5} \mathrm{M}\right.$ dose only) inhibited tube formation. Statistically significant comparisons $\left({ }^{*} P<0.05\right)$ Scale bar $=500 \mu \mathrm{m}$.

up-regulated, and increasing levels of basal and stimulated cGMP are observed in response to endogenous NO or exogenous stimulation of sGC.

We further assessed whether chronic L-NAME treatment could differentially regulate cGMP levels at late stages of differentiation. To do so, we differentiated ESCs for 14 days in the presence of L-NAME $\left(10^{-5} \mathrm{M}\right)$ or vehicle control and measured cGMP levels after stimulating with A23187 (to stimulate endogenous NO synthesis) or the exogenous activators of sGC, SNAP, or BAY41. Chronic L-NAME treatment significantly reduced the levels of cGMP production after A23187 stimulation by $58 \% \pm 14 \%$, confirming that A23187 increased cGMP by activating endogenous NOS $(P<0.05$, Fig. 6E). L-NAME also blunted the increase in cGMP induced by BAY41, which is consistent with the effect of endogenous NO to potentiate the effect of BAY41. The potentiation of the effect of BAY41 by A23187 was also inhibited by L-NAME (by $33 \% \pm 6 \%, P<0.05$ ), which is consistent with the effect of A23187 to increase NO synthesis. The effect of SNAP to increase cGMP levels (either alone or in the presence of BAY41)
A

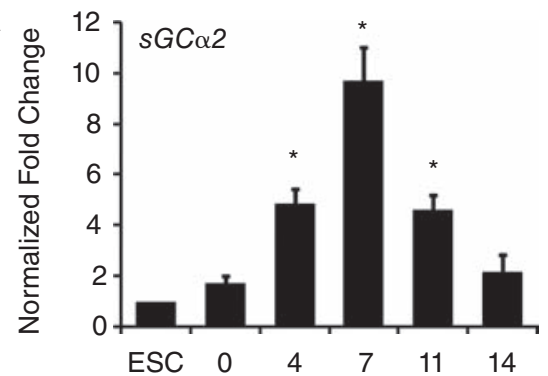

C

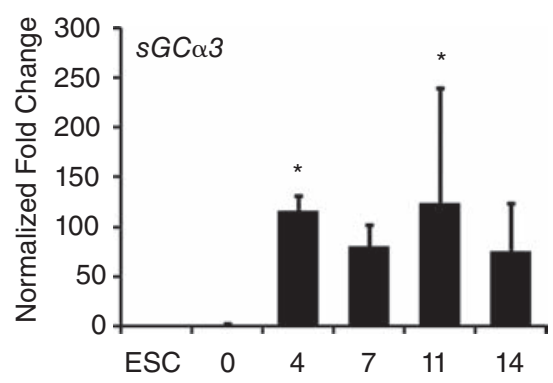

E

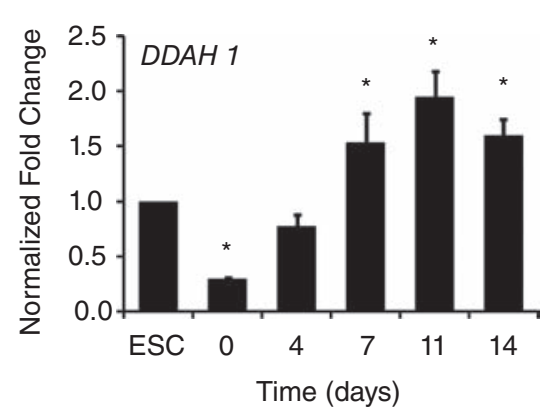

B

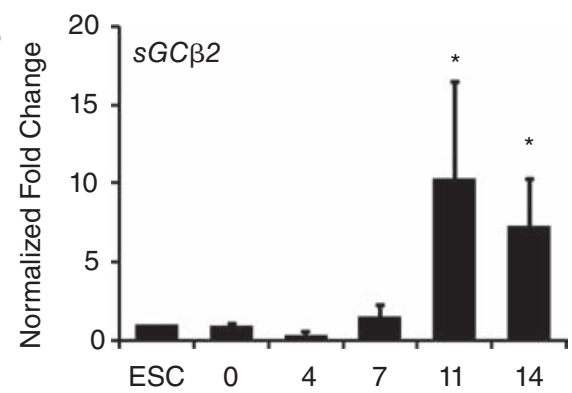

D

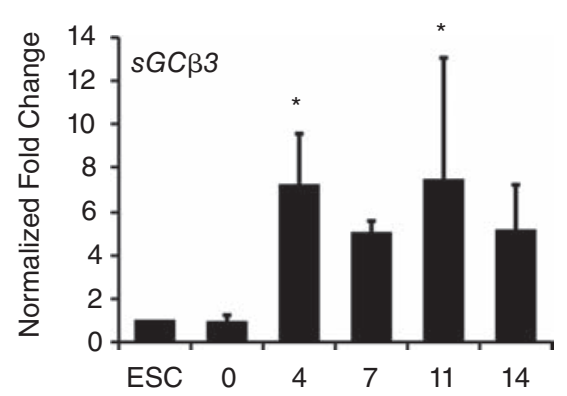

$\mathbf{F}$

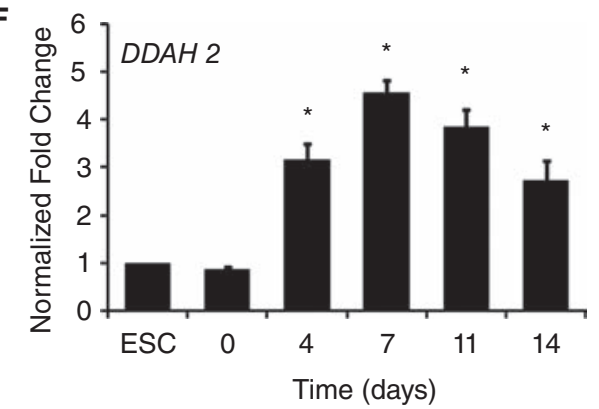

FIG.4. Time course of soluble guanylyl cyclase (sGC) and dimethylarginine dimethylaminohydrolase $(D D A H)$ gene expression during endothelial differentiation. Embryonic stem cells (ESCs) were cultured in differentiation media for 14 days and assayed at specified time points by quantitative PCR. Data shown is normalized to $18 S$ housekeeping gene and expressed as fold changes relative to ESC group. *Statistically significant compared to ESCs group, $P<0.05(n=3)$. 
FIG. 5. Immunofluorescence staining depicting soluble guanylyl cyclase (sGC) $\alpha_{3}$ and $\mathrm{sGC} \beta_{3}$ expression during the course of endothelial differentiation in embryonic stem cells. Immunofluorescence staining is shown for (A) sGC $\alpha_{3}$ and (B) $\mathrm{sGC} \beta_{3}$, with corresponding Hoechst 33342 nuclear dye. (C) After 14 days of differentiation, the cells were immunofluorescently stained for both CD144 and $\mathrm{sGC} \alpha_{3}$ or $\mathrm{sGC} \beta_{3}$, with corresponding Hoechst 33342 nuclear dye. Scale bar $=200$ $\mu \mathrm{m}$ (A and B); $25 \mu \mathrm{m}(\mathbf{C})$. Color images available online at www.liebertonline.com/scd.

\section{A \\ $\mathrm{sGC} \alpha 3$ \\ $\mathrm{sGC} \alpha 3 /$ \\ Hoechst}
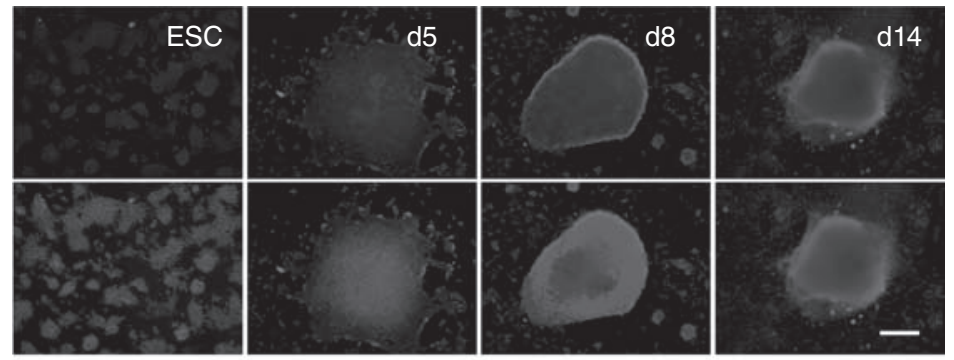

B

B
sGC $\beta 3$
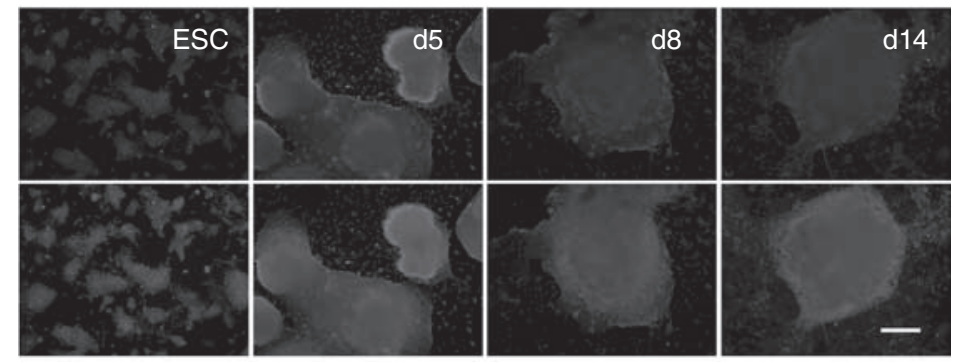

C
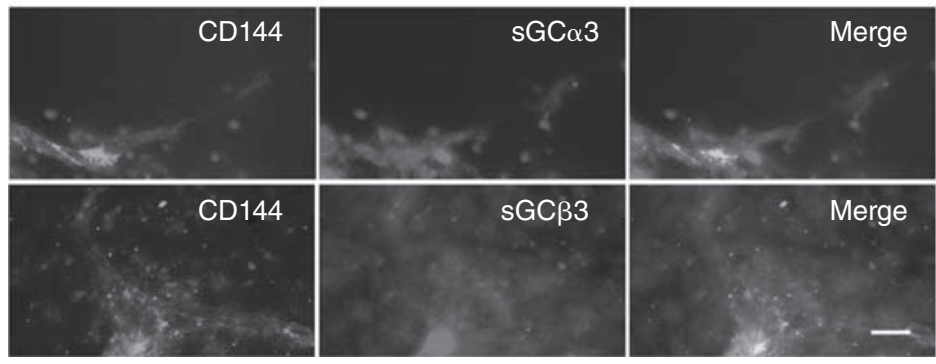

was not affected by L-NAME, consistent with the fact that SNAP is an exogenous NO donor and not dependent upon NOS activity. These observations confirm that by day 14 , elements of the NOS pathway are active, and can generate cGMP in response to endogenous or exogenous NO.

\section{Discussion}

The salient findings of this study are that (1) ESCs possess key elements of the NO-signaling machinery, but this pathway is functionally inactive in ESCs; (2) with differentiation, there is an increase in the expression of endothelial surface markers, eNOS, and subunits of sGC, in association with increasing activity of the NO-signaling machinery as assessed by cGMP production; and (3) chronic inhibition of NOS has no effect early in differentiation, but by 14 days of differentiation modestly inhibits the expression of endothelial surface markers CD144, FLK1, and eNOS, as well as the formation of endothelial tubes.

The NOS pathway is ubiquitous in the adult mammal, with endothelial, neuronal, and inducible NOS isoforms playing critical roles in every organ system, in particular cardiovascular homeostasis, thrombosis, neurotransmission, and immunity [24]. With respect to vascular reactivity and structure, NO plays a dominant role as a vasodilation factor that also potently inhibits vascular smooth muscle proliferation, leukocyte infiltration, and platelet adherence and aggregation [25]. Furthermore, NO is a powerful agonist of EC proliferation and migration. There is also a reciprocal reinforcing relationship between $\mathrm{NO}$ and vascular endothelial growth factor (VEGF), as VEGF stimulates NO synthesis, and NO increases VEGF expression [16]. Inhibition of NOS substantially reduces growth factor-induced angiogenesis, whereas enhancing endogenous NO synthesis promotes angiogenesis $[17,26]$. In particular, L-NAME blocks VEGF-induced cGMP production in human umbilical venous ECs when cultured in collagen gels [27]. Whereas L-NAME is not metabolized by cells, asymmetrical dimethylarginine (ADMA) is an endogenous NOS inhibitor that is degraded by DDAH. Plasma ADMA is elevated in vascular diseases and associated disorders, due in part to impairment in DDAH activity [28-30].

In view of the importance of NO in mammalian physiology and particularly its role in adult angiogenesis, it is somewhat surprising that embryonic lethality is not observed with homozygous eNOS deficiency. Even animals deficient for all 3 NOS isoforms may survive to adulthood (although hypertensive, infertile, and with nephrogenic diabetes insipidus) [31]. Our results provide a partial explanation for this phenomenon. We observe that early in differentiation, although the key elements of the NOS pathway are present, they are functionally inactive. Accordingly, other signaling systems for endothelial growth must be primary early in development. In the adult mammal, other signaling molecules such as prostacyclin, epoxyeicosatrienoic acids, carbon monoxide, hydrogen peroxide, adrenomedullin, or C-type natriuretic peptide provide for redundancy, and become more evident when NOS is inhibited. It is possible that one or more of these signaling molecules play an important role in vascular development.

Previous studies have surveyed the differential expression of NO-signaling components during cardiac differentiation of ESCs. When murine embryoid bodies are incubated with the NOS antagonist N-monomethyl-L-arginine (L-NMMA) 

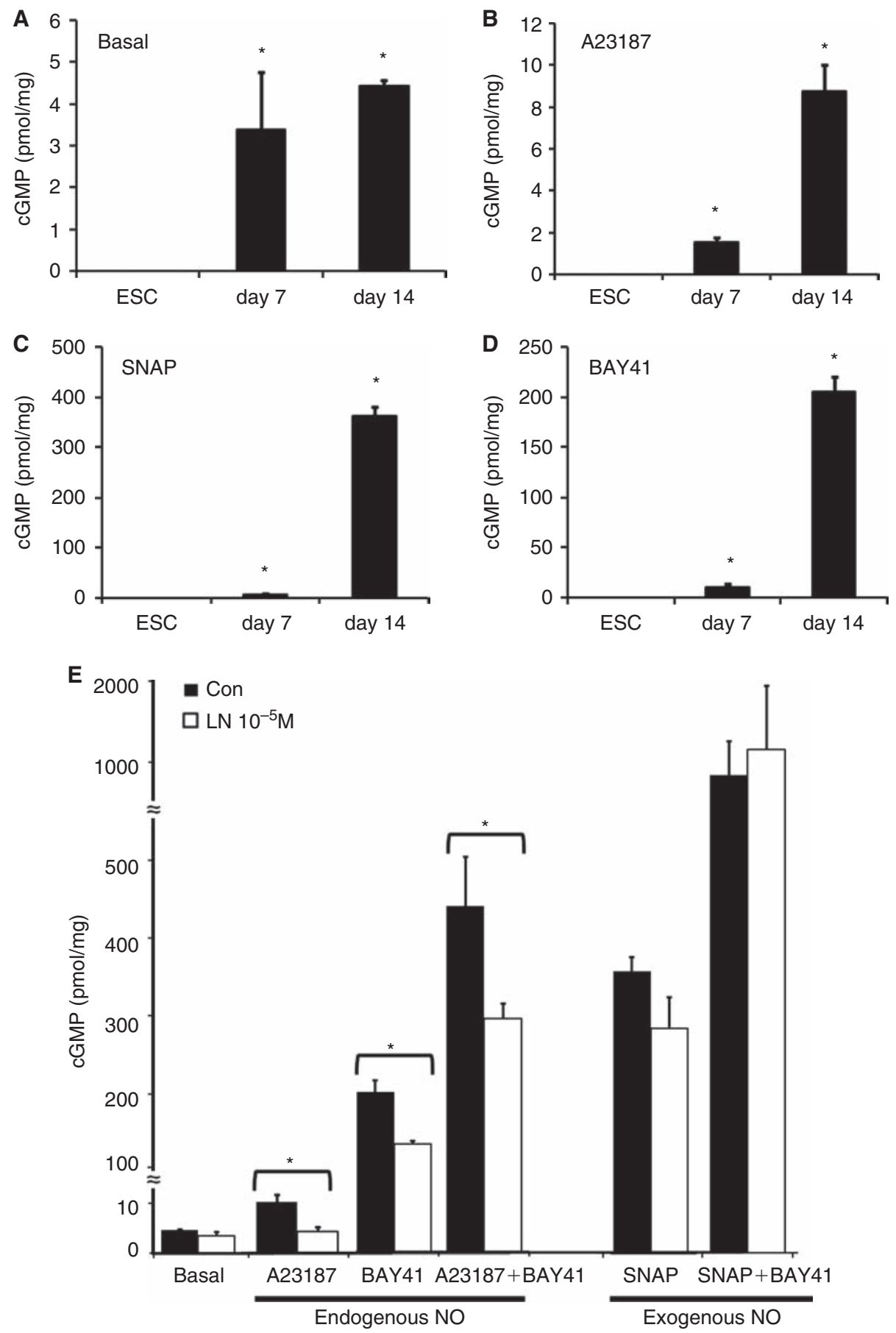

FIG. 6. Effect of endogenous or exogenous activators of nitric oxide (NO) on cyclic guanosine monophosphate (cGMP) activity. At different time points of differentiation, cell lysates were assayed for cGMP production after (A) no treatment (basal), (B) A23187, (C) SNAP, or (D) BAY41 stimulation. (E) After 14 days of differentiation in media containing NG-nitroarginine methyl ester (L-NAME) $\left(10^{-5} \mathrm{M}\right)$, cells were stimulated with activators of NO release, and lysates were assayed for cGMP. Asterisk with brackets indicates statistically significant comparisons, and asterisk without brackets indicate statistically significant when compared to embryonic stem cells (ESCs) $(P<0.05)$.

or L-nitroarginine, the number of EB-derived cardiomyocytes is not affected, but fibrillogenesis in the cardiomyocytes is reduced [32]. This effect is mimicked by ODQ (an antagonist of soluble guanylate cyclase). These observations are consistent with those of Krumenacker et al. who showed an increase in expression of $s G C \alpha_{1}$ and $s G C \beta_{1}$ expression during cardiac differentiation of murine ESCs [11]. Mujoo et al. also demonstrated an increase of $s G C$ subunits during the course of differentiation of human ESCs for 14 days, but the authors reported a progressive decrease in $s G C \beta_{2}$ mRNA from day 0 to day 14 [10]. These observed differences may be attributed to differences in the ESC species and strains, 
as well as varying induction media formulations. In a recent study of human and mouse ESCs, NO donors and sGC activators could increase the mRNA expression of cardiacspecific genes myosin light chain and Nkx2.5, whereas NOS inhibitors decreased their mRNA expression [9].

A limitation of the current study is the inherent heterogeneity of the population of differentiating cells, which includes ESC-ECs. Nevertheless, this mixed population can respond to pharmacological agents and provide useful biochemical and signaling cues to better understand the role of NO in ESC differentiation.

In summary, we have demonstrated that NO-signaling elements are present in ESCs, although the NOS pathway is functionally inactive until later in differentiation. Pharmacological inhibition of NOS has no observed effect early in differentiation on endothelial gene expression and surface markers, but later in differentiation causes a modest reduction of these genes and markers, as well as a modest impairment of endothelial tube-like formation. Our studies indicate that later in differentiation NO plays a role in endothelial development and function, but other signaling pathways must play a greater role early in development.

\section{Acknowledgments}

This study was supported by grants from the National Institutes of Health (RO1 HL-75774, R01 CA098303, R21 HL085743, 1K12 HL087746), the California Tobacco Related Disease Research Program of the University of California (1514RT-0169), the American Heart Association (0970036N) and the California Institute for Regenerative Medicine (RS1-00183), and the Stanford Cardiovascular Institute. N.F.H. was supported by a fellowship from the American Heart Association.

\section{Author Disclosure Statement}

No competing financial interests exist.

\section{References}

1. Thomson JA, J Itskovitz-Eldor, SS Shapiro, MA Waknitz, JJ Swiergiel, VS Marshall and JM Jones. (1998). Embryonic stem cell lines derived from human blastocysts. Science 282:1145-1147.

2. Reubinoff BE, MF Pera, CY Fong, A Trounson and A Bongso. (2000). Embryonic stem cell lines from human blastocysts: Somatic differentiation in vitro. Nat Biotechnol 18:399-404.

3. Behfar A, LV Zingman, DM Hodgson, JM Rauzier, GC Kane, A Terzic and M Pucéat. (2002). Stem cell differentiation requires a paracrine pathway in the heart. FASEB J 16:1558-1566.

4. Levenberg S, JS Golub, M Amit, J Itskovitz-Eldor and R Langer. (2002). Endothelial cells derived from human embryonic stem cells. Proc Natl Acad Sci USA 99:4391-4396.

5. Yamashita J, H Itoh, M Hirashima, M Ogawa, S Nishikawa, T Yurugi, M Naito, K Nakao and S Nishikawa. (2000). Flk1positive cells derived from embryonic stem cells serve as vascular progenitors. Nature 408:92-96.

6. Yang L, MH Soonpaa, ED Adler, TK Roepke, SJ Kattman, M Kennedy, E Henckaerts, K Bonham, GW Abbott, RM Linden, LJ Field and GM Keller. (2008). Human cardiovascular progenitor cells develop from a KDR+ embryonic-stem-cell-derived population. Nature 453:524-528.

7. Moncada S and A Higgs. (1993). The L-arginine-nitric oxide pathway. N Engl J Med 329:2002-2012.

8. Boerrigter G and JC Burnett Jr. (2009). Soluble guanylate cyclase: not a dull enzyme. Circulation 119:2752-2754.
9. Mujoo K, VGSharin, NSBryan, JSKrumenacker, CSloan, SParveen, LE Nikonoff, AY Kots and F Murad. (2008). Role of nitric oxide signaling components in differentiation of embryonic stem cells into myocardial cells. Proc Natl Acad Sci USA 105:18924-18929.

10. Mujoo K, JS Krumenacker, Y Wada and F Murad. (2006). Differential expression of nitric oxide signaling components in undifferentiated and differentiated human embryonic stem cells. Stem Cells Dev 15:779-787.

11. Krumenacker JS, S Katsuki, A Kots and F Murad. (2006). Differential expression of genes involved in cGMP-dependent nitric oxide signaling in murine embryonic stem (ES) cells and ES cell-derived cardiomyocytes. Nitric Oxide 14:1-11.

12. Danalache BA, J Paquin, W Donghao, R Grygorczyk, JC Moore, CL Mummery, J Gutkowska and M Jankowski. (2007). Nitric oxide signaling in oxytocin-mediated cardiomyogenesis. Stem Cells 25:679-688.

13. Lee TC, YD Zhao, DW Courtman and DJ Stewart. (2000). Abnormal aortic valve development in mice lacking endothelial nitric oxide synthase. Circulation 101:2345-2348.

14. Feng Q, W Song, X Lu, JA Hamilton, M Lei, T Peng and SP Yee. (2002). Development of heart failure and congenital septal defects in mice lacking endothelial nitric oxide synthase. Circulation 106:873-879.

15. Niebauer J, J Dulak, JR Chan, PS Tsao and JP Cooke. (1999). Gene transfer of nitric oxide synthase: effects on endothelial biology. J Am Coll Cardiol 34:1201-1207.

16. Dulak J, A Józkowicz, A Dembinska-Kiec, I Guevara, A Zdzienicka, D Zmudzinska-Grochot, I Florek, A Wójtowicz, A Szuba and JP Cooke. (2000). Nitric oxide induces the synthesis of vascular endothelial growth factor by rat vascular smooth muscle cells. Arterioscler Thromb Vasc Biol 20:659-666.

17. Jacobi J, K Sydow, G von Degenfeld, Y Zhang, H Dayoub, B Wang, AJ Patterson, M Kimoto, HM Blau and JP Cooke. (2005). Overexpression of dimethylarginine dimethylaminohydrolase reduces tissue asymmetric dimethylarginine levels and enhances angiogenesis. Circulation 111:1431-1438.

18. Cao F, KE van der Bogt, A Sadrzadeh, X Xie, AY Sheikh, H Wang, AJ Connolly, RC Robbins and JC Wu. (2007). Spatial and temporal kinetics of teratoma formation from murine embryonic stem cell transplantation. Stem Cells Dev 16:883-891.

19. Shetzline SE, R Rallapalli, KJ Dowd, S Zou, Y Nakata, CR Swider, A Kalota, JK Choi and AM Gewirtz. (2004). Neuromedin U: a Myb-regulated autocrine growth factor for human myeloid leukemias. Blood 104:1833-1840.

20. Livak KJ and TD Schmittgen. (2001). Analysis of relative gene expression data using real-time quantitative PCR and the 2(-Delta Delta C(T)) Method. Methods 25:402-408.

21. Huang NF, S Patel, RG Thakar, J Wu, BS Hsiao, B Chu, RJ Lee and S Li. (2006). Myotube assembly on nanofibrous and micropatterned polymers. Nano Lett 6:537-542.

22. Caiado F, C Real, T Carvalho and S Dias. (2008). Notch pathway modulation on bone marrow-derived vascular precursor cells regulates their angiogenic and wound healing potential. PLoS ONE 3:e3752.

23. Budworth J, S Meillerais, I Charles and K Powell. (1999). Tissue distribution of the human soluble guanylate cyclases. Biochem Biophys Res Commun 263:696-701.

24. Bryan NS, K Bian and F Murad. (2009). Discovery of the nitric oxide signaling pathway and targets for drug development. Front Biosci 14:1-18.

25. Cooke JP. (2003). Flow, NO, and atherogenesis. Proc Natl Acad Sci USA 100:768-770.

26. Jang JJ, HK Ho, HH Kwan, LF Fajardo and JP Cooke. (2000). Angiogenesis is impaired by hypercholesterolemia: role of asymmetric dimethylarginine. Circulation 102:1414-1419.

27. Papapetropoulos A, G García-Cardeña, JA Madri and WC Sessa. (1997). Nitric oxide production contributes to the angiogenic properties of vascular endothelial growth factor in human endothelial cells. J Clin Invest 100:3131-3139. 
28. Cooke JP. (2004). Asymmetrical dimethylarginine: the Uber marker? Circulation 109:1813-1818.

29. Lin KY, A Ito, $\mathrm{T}$ Asagami, PS Tsao, $\mathrm{S}$ Adimoolam, M Kimoto, $\mathrm{H}$ Tsuji, GM Reaven and JP Cooke. (2002). Impaired nitric oxide synthase pathway in diabetes mellitus: role of asymmetric dimethylarginine and dimethylarginine dimethylaminohydrolase. Circulation 106:987-992.

30. Böger RH, SM Bode-Böger, A Szuba, PS Tsao, JR Chan, O Tangphao, TF Blaschke and JP Cooke. (1998). Asymmetric dimethylarginine (ADMA): a novel risk factor for endothelial dysfunction: its role in hypercholesterolemia. Circulation 98:1842-1847.

31. Morishita T, M Tsutsui, H Shimokawa, K Sabanai, H Tasaki, O Suda, S Nakata, A Tanimoto, KY Wang, Y Ueta, Y Sasaguri, Y Nakashima and N Yanagihara. (2005). Nephrogenic diabetes insipidus in mice lacking all nitric oxide synthase isoforms. Proc Natl Acad Sci USA 102:10616-10621.
32. Bloch W, BK Fleischmann, DE Lorke, C Andressen, B Hops, J Hescheler and K Addicks. (1999). Nitric oxide synthase expression and role during cardiomyogenesis. Cardiovasc Res 43:675-684.

Address correspondence to: Dr. John P. Cooke Division of Cardiovascular Medicine Stanford University 300 Pasteur Drive Stanford, CA 94305-5406

E-mail: john.cooke@stanford.edu

Received for publication October 22, 2009

Accepted after revision January 9, 2010

Prepublished on Liebert Instant Online January 11, 2010 
